\section{Effect of the neridronate on RANKL release in differentiated primary human osteoblasts}

\author{
Vanessa Nicolin, ${ }^{1}$ Roberto Valentini, ${ }^{2}$ \\ Roberta Bortul, ${ }^{1}$ Giovanni Fancellu ${ }^{2}$ \\ ${ }^{1}$ Clinical Department of Biomedicine, \\ section of Molecular Biology, University \\ of Trieste; ${ }^{2}$ Clinical Department \\ of Specialized Surgical Sciences, \\ Biomaterials and Bioimplants, U.C.O. \\ of Clinical Orthopedics and Traumatology, \\ University of Trieste, Italy
}

\section{Abstract \\ Bisphosphonates are important inhibitors of bone resorption and widely used clinically to treat osteoporosis, metabolic bone diseases and other orthopaedic disorders . Inhibiting osteoclasts via the mevalonate pathway is rec- ognized as the primary mechanism of its inhibitory action. Recent evidence suggests that bisphosphonates may regulate essential signaling molecules involved in osteoclastoge- nesis such as RANKL (receptor activator of NF- $\mathrm{kB}$ ligand) which are synthesized by osteoblasts. In this report we have investigated into the neridronate-osteoblast interactions in modulating essential signaling molecule such as RANKL.}

Bisphosphonates (BPs) are stable pyrophosphate analogs that have been successfully used to treat postmenopausal and glucocorticoidinduced osteoporosis, as well as Paget's disease of bone, metastatic and osteolytic bone disease, and hypercalcemia of malignancy. ${ }^{1}$ Bisphosphonates are analogues of endogenous pyrophosphates in which a carbon atom replaces the central atom of oxygen. As described by Font et al., ${ }^{2}$ while the central ring is essential for binding hydroxyapatite, the lateral chains (R1 and R2) are variable and can either 60 contain a terminal amino chain or a cyclic nitrogen chain. Of the two side-chains, it is understood that the R1 side-chain is usually a hydroxyl group that enhances the affinity of the compound to bone. ${ }^{3,4}$ The potency of the compound as an anti-resorptive is determined by the variations in the structure and conformation of the R2 side-chain. ${ }^{5}$ Based on these side-chains, bisphosphonates can be divided into two main groups: nitrogencontaining/amino bisphosphonates (N-BPs) (Alendronate, Risedronate, Pamidronate, Zoledronate, Neridronate, Ibandronate) and non-nitrogen containing bisphosphonates
(Etidronate, Tiludronate). There is good evidence that the inhibition of bone resorption in patients receiving BPs is caused by the inhibition of osteoclast (OC) precursor proliferation, differentiation, ${ }^{6,7}$ and the induction of apoptosis in mature 0Cs. ${ }^{7}$ Significant strides have been made in understanding the mechanism of action of bisphosphonates. Inhibiting osteoclasts via the mevalonate pathway is recognized as the primary mechanism of its inhibitory action. ${ }^{7}$ Recent evidence suggests that bisphosphonates may not only reduce osteoclast maturation and function directly but also act indirectly by activating osteoblast-stromal cells to synthesize mediators that interfere with osteoclastogenesis. ${ }^{8,9}$ Bisphosphonates also regulate essential signaling molecules involved in osteoclastogenesis such as osteoclast situlating factors. ${ }^{10}$ The development of OCs is controlled by osteoclastogenic factors synthesized by osteoblasts (OBs), including RANKL and osteoprotegerin (OPG). RANKL (also known as TRANCE/OPGL/ODF) plays a pivotal role in osteoclastogenesis by providing an essential signal to $\mathrm{OC}$ progenitors through the membrane-anchored receptor RANK. ${ }^{11}$ Signal transduction through RANK leads to OC differentiation and functional activation. This signaling pathway can be disrupted by a naturally occurring decoy receptor for RANKL, termed osteoprotegerin (OPG), which blocks the interaction between RANKL and RANK. ${ }^{11,12}$ Bone marrow OB/stromal cells secrete both RANKL and OPG, and $\mathrm{OC}$ formation is determined principally by the relative ratio of RANKL to OPG in the bone marrow (BM) microenvironment. Recent evidence suggests that some bisphosphonates also regulate essential signaling molecules involved in osteoclastogenesis such as RANKL (receptor activator of NF-kB ligand) which are synthesized by osteoblasts, and bisphosphonates also modulate OPG (Osteoprotegerin), a decoy receptor absorbing RANKL and preventing RANK activation. ${ }^{13}$ With this aim we have investigated the effect of neridronate on primary human osteoblast cell line producing RANKL. To analyze the effects of neridronate on human osteoblasts cells, osteoblasts were plated in each well of 6 -well plates with $2 \mathrm{~mL}$ of $\alpha$-MEM supplemented with $10 \% \mathrm{FBS}, 1 \%$ penicillin/streptomycin and 1\% L-glutamine, incubated at $37^{\circ} \mathrm{C}$ and equilibrated in $5 \% \mathrm{CO}_{2}$ in air. ${ }^{13}$ After $24 \mathrm{~h}$ incubation, fresh media was supplemented with $10 \mathrm{mM} \beta$-glycerophosphate, $0.1 \mathrm{mM}$ ascorbic acid, and $10{ }^{-5} \mathrm{M}$ of neridronate or controls ([10-8 $\left.{ }^{8} \mathrm{M}\right]$ Vitamin D3). ${ }^{13}$ Cells were cultured for an additional 2, 4 and 7 days after administration. In control untreated osteoblasts, RANKL protein level peaked at 4 day, phenomenon that is attributed to osteoblast differentiation while compared to the treated cells, at all time periods, neridronate resulted in a down-regulation of
Correspondence: Nicolin Vanessa, University of Trieste, Clinical Department of Biomedicine, section of Molecular Biology, via Manzoni, 16 34138 Trieste. E-mail: nicolin@units.it

Key words: receptor activator of NF-kB ligand, human osteoblasts, neridronate.

Acknowledgements: the authors thank ABIOGEN PHARMA for supporting the research.

Received for publication: 29 November 2010. Revision received: 3 March 2011.

Accepted for publication: 7 March 2011.

This work is licensed under a Creative Commons Attribution 3.0 License (by-nc 3.0).

() Copyright V. Nicolin et al., 2011

Licensee PAGEPress, Italy

Drugs and Therapy Studies 2011; 1:e3

doi:10.4081/dts.2011.e3

RANKL expression (Figurel a,b). The decrease of RANKL expression (Figure 2) is also evidenced by immunohistochemistry in situ. ${ }^{14}$ As demonstrated in Figure 2a untreated osteoblast cells revealed a high level of RANKl cytoplasmatic expression while treated cells presented a downregulation of protein expression (Figure 2b). The decrease of transmembrane RANKL expression (Figure 2b) evidenced by immunohistochemistry unaccompanied by a commensurate loss in total RANKL protein (Figure 1b), suggested that nitrogen-containing bisphosphonates may mediate their effects posttrascriptionally. Our results should be consid-

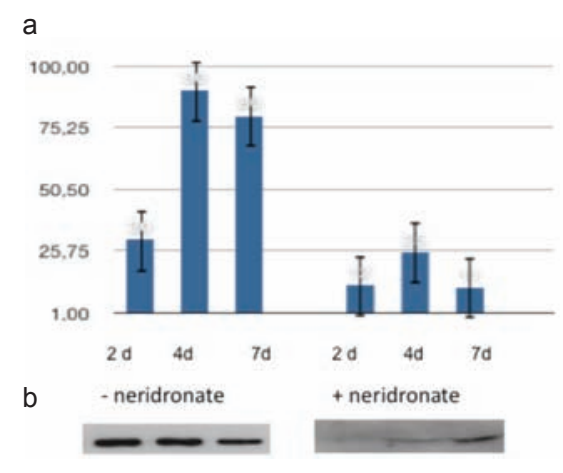

Figure 1. (a) Statistical analysis carried out using densitometry measurements showing that human primary osteoblast cells untreated expressed high level of RANKL in comparison with osteoblasts treated with Neridronate. This data are confirmed by the results obtained by immunoblotting. (b) The results were evaluated by using analysis of variance with subsequent comparisons by Student's t-test for paired or non-paired data, as appropriate. Statistical significance was defined as $\mathbf{P}<\mathbf{0 . 0 5}$. Values are reported as means \pm standard deviation (Microsoft, Excel). 
ered in the context of several previous studies, which have reported contrasting and sometimes opposite role of bisphophonates during the osteoclastogenesis process. Recent evidence suggests that bisphosphonates may not only reduce osteoclast maturation and function
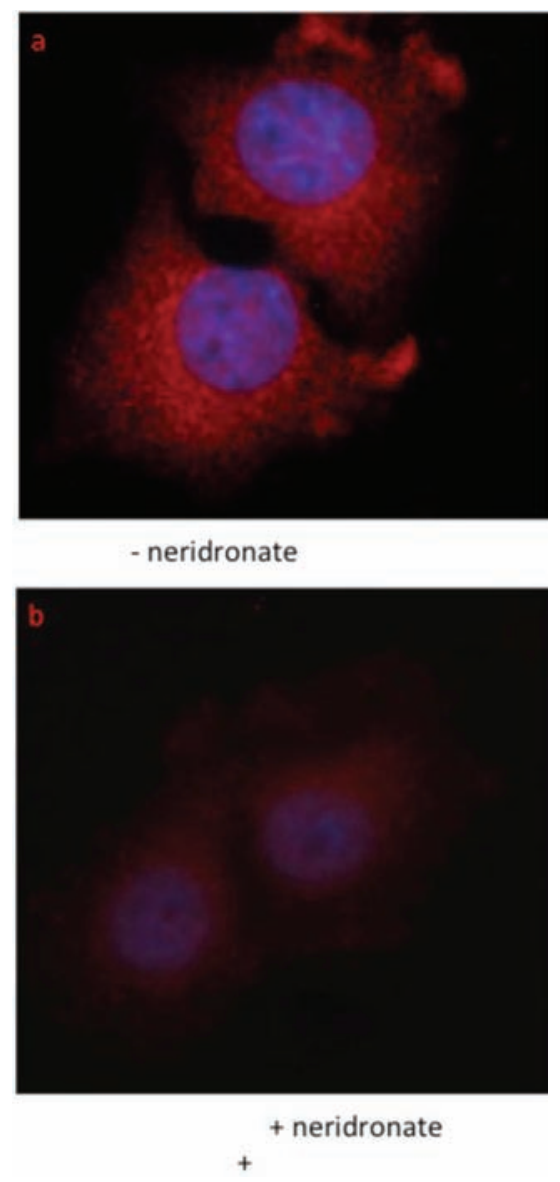

Figure 2. Immunolabelling for RANK-ligand shows cytoplasmic localization of RANKL scattered as many dots over cytoplasmic region of human osteoblast cells. On the left side osteoblast cells were untreated and revealed a high presence of RANKI- protein on the cytoplasmatic region. On the contrary, on the right side osteoblasts cells treated with Neridronate showed a complete downregulation of the RANKL protein. directly but also act indirectly by activating osteoblast-stromal cells to synthesize mediators that interfere with osteoclastogenesis ${ }^{10-12}$ decreasing RANKLm-RNA expression in a rat osteoblast cell line. On the contrary and Kim and coworkers ${ }^{16}$ have presented data that do not agree with our findings. These groups reported that bisphosphonates do not impact RANKL and OPG gene expression. ${ }^{15}$ The discrepancy in their data may be due to different cell line used infact, Kim et al. used mouse osteoblast-like cells not primary human cells. ${ }^{15}$ In conclusion aur findings demostrated hat neridronate could impact the expression of RANKI postrascriptionally. The results from this preliminary study lead support the suggestion that the inhibitory effects of neridronic on the inhibition of the RANKL rather than to modulation of its expression.

\section{References}

1. Chambers AF, Groom AC, MacDonald IC. Dissemination and growth of cancer cells in metastatic sites. Nat Rev Cancer 2002; 2:563-72.

2. Font RG, Garcia MLM, Martinez JMO. Osteochemonecrosis of the jaws due to bisphosphonate treatments. Update. Med Oral Patol Cir Bucal 2008;13:E318-24.

3. Widler L, Jaeggi KA, Glatt M, et al. Highly potent geminal bisphosphonates. From pamidronate disodium (aredia) to zoledronic Acid (zometa). J Med Chem 2002;45:3721-38.

4. Rogers MJ, Watts DJ, Russell RG. Inhibitory effects of bisphosphonates on growth of amoebae of the cellular slime mold Dictyostelium discoideum. J Bone Miner Res 1994;9:1029-39.

5. Russell RG, Rogers MJ, Frith JC, et al. The pharmacology of bisphosphonates and new insights into their mechanisms of action. J Bone Miner Res 1999;14:53-65

6. Hughes DE, MacDonald BR, Russell RG, Gowen M. Inhibition of osteoclast-like cell formation by bisphosphonates in long- term cultures of human bone marrow. J Clin Invest 1989;83:1930-5.

7. Schmidt A, Rutledge SJ, Endo N, et al. Protein-tyrosine phosphatase activity regulates osteoclast formation and function: Inhibition by alendronate. Proc Natl Acad Sci USA 1996; 93:3068-73.

8. Hsu H, Lacey DL, Dunstan CR, et al. Tumor necrosis factor receptor family member RANK mediates osteoclast differentiation and activation induced by osteoprotegerin ligand. Proc Natl Acad Sci USA 1999;96: 3540-45

9. Yasuda H, Shima N, Nakagawa N, et al.. A novel molecular mechanism modulating osteoclast differentiation and function. Bone 1999;25:109-13.

10. Yasuda H, Shima N, Nakagawa N, et al. Osteoclast differentiation factor is a ligand for osteoprotegerin/osteoclastogenesisinhibitory factor and is identical to TRANCE/RANKL. Proc Natl Acad Sci USA 1998;95:3597-602.

11. Yasuda H, Shima N, Nakagawa N, et al. Anovel molecular mechanism modulating osteoclast differentiation and function. Bone 1999;25:109-13.

12. Yasuda H, Shima N, Nakagawa N, et al. Identity of osteoclastogenesis inhibitory factor (OCIF) and osteoprotegerin (OPG): A mechanism by which OPG/OCIF inhibits osteoclastogenesis in vitro. Endocrinology 1998;139:1329-37.

13. Im GI, Qureshi SA, Kenney J, et al. Osteoblast proliferation and maturation by bisphosphonates. Biomaterials 2004;25:4 105-15.

14. Nicolin V, Bortul R, Bareggi R, et al. Breast adenocarcinoma MCF-7 cell line induces spontaneous osteoclastogenesis via a RANK-ligand-dependent pathway. Acta Histochem 2008;110:388-96.

15. Kim YH, Kim GS, Jeong-Hwa B. Inhibitory action of bisphosphonates on bone resorption does not involve the regulation of RANKL and OPG expression. Exp Mol Med 2002;34:145-51. 